\title{
Design and Fabrication of the EM Wave Absorber with Excellent Characteristics for ETC System
}

\author{
Dong Il Kim $\cdot$ Dong Soo Choi $\cdot$ Dong Han Choi $\cdot$ Do Yeol Kim
}

\begin{abstract}
In this paper, the EM wave absorber for ETC system was designed and fabricated. We fabricated several samples in different composition ratios of flaked sendust and CPE(Chlorinated Polyethylene). Absorption abilities were simulated in accordance with different thicknesses of the prepared absorbers and changed complex relative permittivity and permeability according to composition ratio. The optimized mixing ratio of flaked sendust and CPE was found as 60 : 40 wt. $\%$ by experiments and simulation. Then the EM wave absorber was fabricated and tested using the simulated data. As a result, the developed EM wave absorber has the thickness of $2.75 \mathrm{~mm}$ and absorption ability was 22.5 $\mathrm{dB}$ in the case of normal incidence at $5.8 \mathrm{GHz}$. Therefore, it was confirmed that the newly developed absorber can be used for ETC system.
\end{abstract}

Key words: ETC, EM Wave Absorber, Flaked Sendust, Permittivity, Permeability, Loss Tangent.

\section{I . Introduction}

Metropolis is faced with serious traffic jam as the advancement of society and development of economy. The government has introduced Intelligent Transport System (ITS) for the solution of these problems. ITS offers the service of various form combining hardware such as road building, traffic, communication technology, and software. Especially, Electric Toll Collection (ETC) system is possible to realize ITS. ETC system is nonstop auto collection system through an application of Dedicated Short Range wireless Communication (DSRC), which uses microwave of the $5.8 \mathrm{GHz}$. ETC is a communication system between a roadside antenna at the toll gate and a vehicular antenna inside the car [1]. When the operation of ETC system was started, communication error was caused in ETC Environment due to undesired electromagnetic (EM) waves from walls, ETC gates, or other possible nearby objects. The optimal use of EM wave absorber is a possible way to mitigate this problem, and several kinds of absorbers are used in various places, such as plate type used for a ceiling of a tollgate [2] or sheet type used for nearby objects as shown in Fig. 1 [3], [4].

In this paper, we attempt to fabricate EM wave absorbers, the thickness of which is less than $3 \mathrm{~mm}$, absorption ability is over $20 \mathrm{~dB}$ at $5.8 \mathrm{GHz}$ for ETC system. The EM wave absorber samples were made of Flaked Sendust with CPE. Then their reflection coeffi-

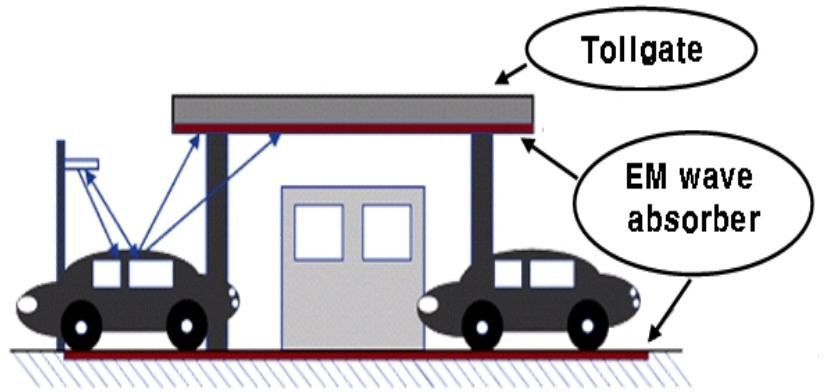

Fig. 1. EM wave absorber used ETC system.

cients were measured. The complex relative permittivity $\left(\varepsilon_{r}=\varepsilon_{r}^{\prime}-j \varepsilon_{r}^{\prime \prime}\right)$ and complex relative permeability $\left(\mu_{r}=\right.$ $\left.\mu_{r}^{\prime}-j \mu_{r}{ }_{r}\right)$ are calculated by the measured data. The EM wave absorber for ETC system is simulated and fabricated based on the simulated design.

\section{Design of EM Wave Absorber}

For an EM wave absorber made of a conductorbacked single layer as shown in Fig. 2, the Return Loss (RL) can be obtained from the equivalent circuit as follows :

$$
R L=-20 \log \left|\frac{z_{\text {in }}-1}{z_{\text {in }}+1}\right| \quad[d B]
$$

where, $z_{i n}$ is the normalized input impedance from the

Manuscript received November 08, 2011 ; Revised January 5, 2012 ; Accepted January 12, 2012. (ID No. 20111108-033J)

Department of Radio Communication Engineering at Korea Maritime University, Busan, Korea.

Corresponding Author : Dong Il Kim (e-mail : dikim@hhu.ac.kr)

This is an Open-Access article distributed under the terms of the Creative Commons Attribution Non-Commercial License (http://creativecommons.org/licenses/ by-nc/3.0) which permits unrestricted non-commercial use, distribution, and reproduction in any medium, provided the original work is properly cited. 


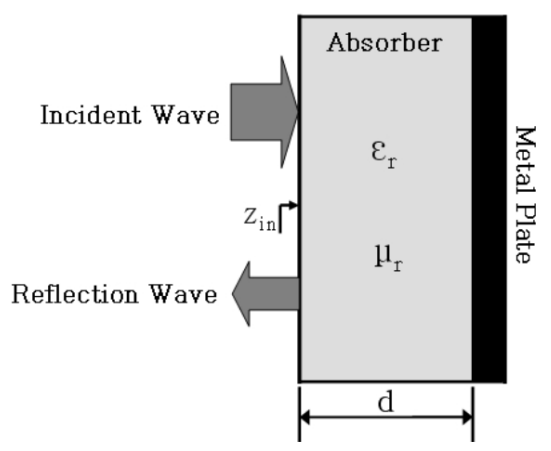

Fig. 2. EM wave absorber in single layer.

surface of absorber.

The normalized input impedance is expressed as equation (2) [5].

$$
z_{i n}=\sqrt{\frac{\mu_{r}}{\varepsilon_{r}} \tanh }\left(j \frac{2 \pi}{\lambda} \sqrt{\varepsilon_{r} \mu_{r}} d\right)
$$

where, $\lambda$ is the wavelength, $d$ is the thickness of the sample, $\varepsilon_{r}$ is the complex relative permittivity, and $\mu_{r}$ is the complex relative permeability. The situation in $R L$ more than $20 \mathrm{~dB}$ ("matching situation") for incidence of an electromagnetic wave is given by [5], [6]

In case of normal incidence wave :

$$
\sqrt{\frac{\boldsymbol{\mu}_{r}}{\varepsilon_{r}} \tanh }\left(j \frac{2 \pi}{\lambda} \sqrt{\varepsilon_{r} \boldsymbol{\mu}_{r}} d\right)=1
$$

In case of oblique incidence wave :

For the TE wave

$$
\frac{\mu_{r} \cos \theta}{\sqrt{\varepsilon_{r} \mu_{r}-\sin ^{2} \theta}} \tanh \left(j \frac{2 \pi d}{\lambda} \sqrt{\varepsilon_{r} \mu_{r}-\sin ^{2} \theta}\right)=1
$$

and for the TM wave

$$
\frac{\sqrt{\varepsilon_{r} \mu_{r}-\sin ^{2} \theta}}{\varepsilon_{r} \cos \theta} \tanh \left(j \frac{2 \pi d}{\lambda} \sqrt{\varepsilon_{r} \mu_{r}-\sin ^{2} \theta}\right)=1
$$

where $\theta$ is the incident angle.

Ideal EM wave absorber can be designed using equation (3), (4), and (5) and it was recognized that the important parameter is the complex relative permittivity, the complex relative permeability, and thickness of EM wave absorber [6].

For the real EM wave absorber design and fabrication, we must various and several samples using the base and raw material of flaked sendust, and CPE, which are fabricated by the composition ratios of the discrete values including the optimum one. In this case, the material constants, i. e., the complex dielectric constants and the complex permeability should be measured. Then, using these constants, the absorber with the best characteristics will be designed by simulations. Finally it will be manufactured and evaluated by experiments.

\section{Preparation and Measurements of Samples}

\section{3-1 Preparation of Samples}

We used flaked sendust as the raw material in order to prepare sheet-type EM wave absorbers. Moreover we processed the flaked sendust powders, which can be manufactured with mechanical forging of spherical Sendust powders using attrition mill to get high value of magnetic permeability and dielectric permittivity. Fig. 3 shows that the SEM images of the Sendust powder. Flaked sendust was mixed with CPE as a binder, and a sheet type absorber was fabricated by using an open roller. The open roller's surface temperature was controlled uniform by $70{ }^{\circ} \mathrm{C}$ during sample preparation because the surface temperature affects the EM wave properties of sheet-type absorbers [7]. In order to investigate the characteristics (material property and Absorption ability etc.) of samples which have a difference composition ratios of materials, EM wave absorber samples were fabricated by the composition ratios of flaked sendust: $\mathrm{CPE}=60: 40 \mathrm{wt} . \%$, flaked sendust: $\mathrm{CPE}=70: 30 \mathrm{wt}$. $\%$, and flaked sendust:CPE=80:20 wt.\%. As CPE portion of EM wave absorber is increasing, the re- lative permeability is decreased. So the optimum ratio of flaked sendust to CPE has been investigated into the EM wave absorber for ETC systems. If CPE portion is too small, however, it is impossible to fabricate EM wave absorber due to stiffness of the absorber.

The fabricating process of an EM wave absorber is shown in Fig. 4.

\section{3-2 Measurements of Samples}

For investigating of the EM wave absorption abilities of the samples, the prepared sheet-type absorbers were punched into a toroidal shape with an inner diameter of $3.05 \mathrm{~mm}$ and an outer diameter of $6.95 \mathrm{~mm}$ (GPC7 ty-

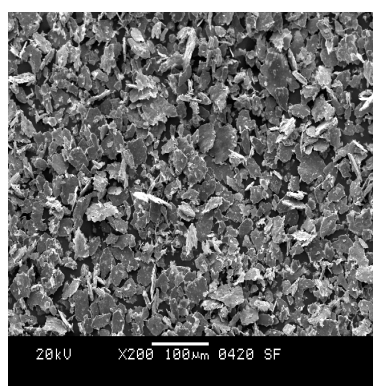

(a) Flaked sendust

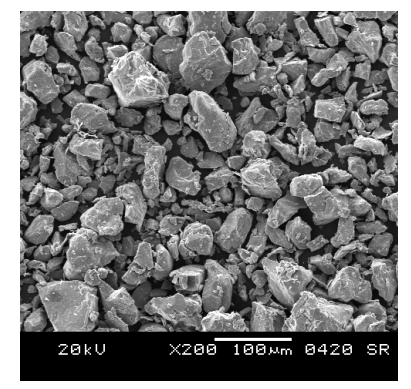

(b) Raw sendust
Fig. 3. SEM of flaked sendust and raw sendust. 


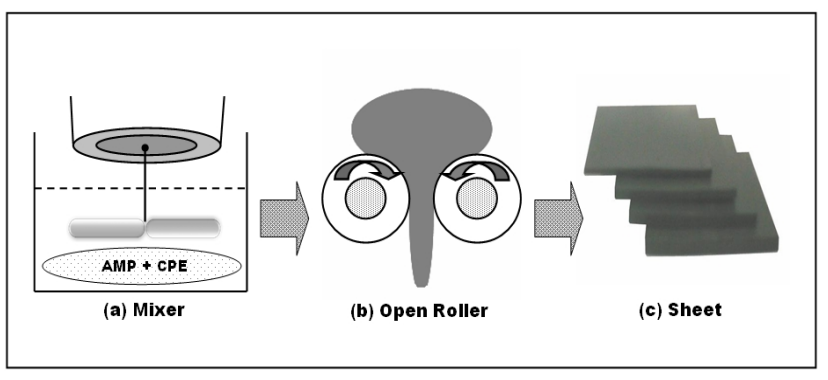

Fig. 4. Fabricating process of an EM wave absorber.

pe). The absorption abilities of the samples were investigated by using network analyzer and gauged by measurement system as shown in Fig. 5 and Fig. 6 presents a photo of the sample holder and sheet-type sample and toroidal shape sample. and the material properties of these samples are calculated from $S$-parameter of samples through $l-2 l$ method [8].

\section{Simulation and Results}

We measured reflection coefficient of the EM wave absorber samples containing flaked sendust with CPE. Fig. 7 shows reflection coefficients of EM wave absorber samples containing difference composition ratio of materials in case of vertical incidence wave. The thickness of the samples are $2 \mathrm{~mm}$ and $4 \mathrm{~mm}$. The sample made of flaked sendust: $\mathrm{CPE}=60: 40$ wt. $\%$ showed the best absorption ability as shown in Fig. 7(a).

Thus, this sample was used for designing the EM wave absorber of ETC system. The optimum absorption ability of flaked sendust: CPE was simulated by using the measured material constants (relative permittivity and relative permeability) of samples and controlling the thickness of absorber based on the eq. (3).

Furthermore, we fabricated EM wave absorbers based on simulation results, thickness is $2.7 \mathrm{~mm}$, absorption

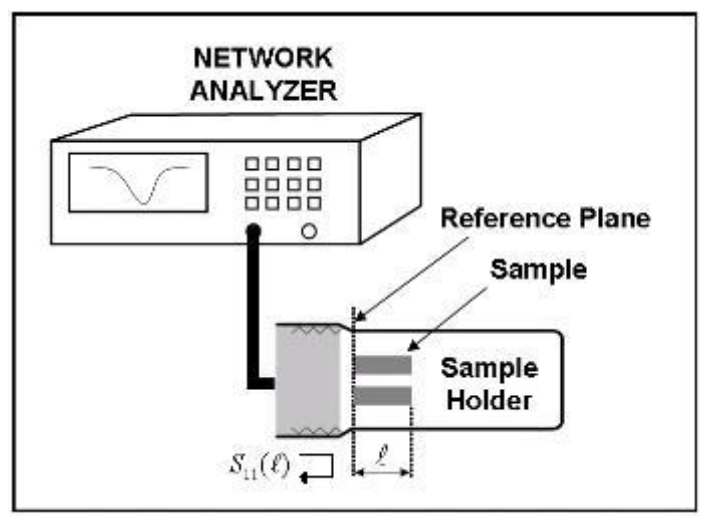

Fig. 5. Measurement system of reflection coefficients (transmission line method).

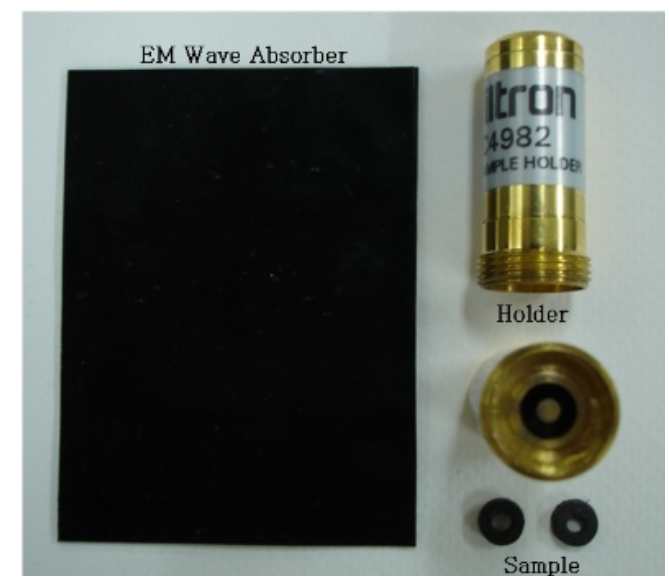

Fig. 6. Photos of EM wave absorber, sample holder, and samples for inserting into the holder.

ability is more than $20 \mathrm{~dB}$ at $5.8 \mathrm{GHz}$ as shown in Fig. 7. The simulated data agree very well with the measured ones.

The reflection coefficents with the incidence angle is usually given by [8]

$$
|S(\Theta)|=\tan ^{2}\left(\frac{\Theta}{2}\right)
$$

In addition, the reflection coefficients for the polarizations of TE and TM waves are given by the left sides of eqs. (4) and (5), respectively. Especially the absorption characteristics for normal incidence were excellent, while the ones for oblique incidence were relatively good.

Firstly, 3 kinds of samples with different ratios of sendust to CPE were fabricated. Then we compared the material constant and regarded that the composition ratio of flaked sendust:CPE $=60: 40$ wt. $\%$ is the best. Secondly, we calculated the optimum thickness of EM wave absorber for ETC system by computer simulation at 5.8 $\mathrm{GHz}$. As a result, we found a few samples with the absorption ability more than $20 \mathrm{~dB}$, and especially the optimized one with the thickness of $2.75 \mathrm{~mm}$ has the most absorption ability of $22.5 \mathrm{~dB}$.

The optimized EM wave absorber with thickness of $2.75 \mathrm{~mm}$ has absorption ability of $22.5 \mathrm{~dB}$ at $5.8 \mathrm{GHz}$ as shown in Fig. 10 where the flaked sendust was used. On the other hand, Fig. 11 shows the absorption of 13 $\mathrm{dB}$ of samples (Amorphous: $\mathrm{CPE}=60: 40 \mathrm{wt}$. \%). The absorption characteristics of the flaked sendust were improved greatly compared with the convetional one.

Fig. 8(a) and (b) show the material costants of the sample with the mixing ratio of flaked sendust:CPE $=60: 40$ wt. $\%$, while Fig. 9 shows the loss tangent of samples with the mixing ratio of flaked sendust:CPE 


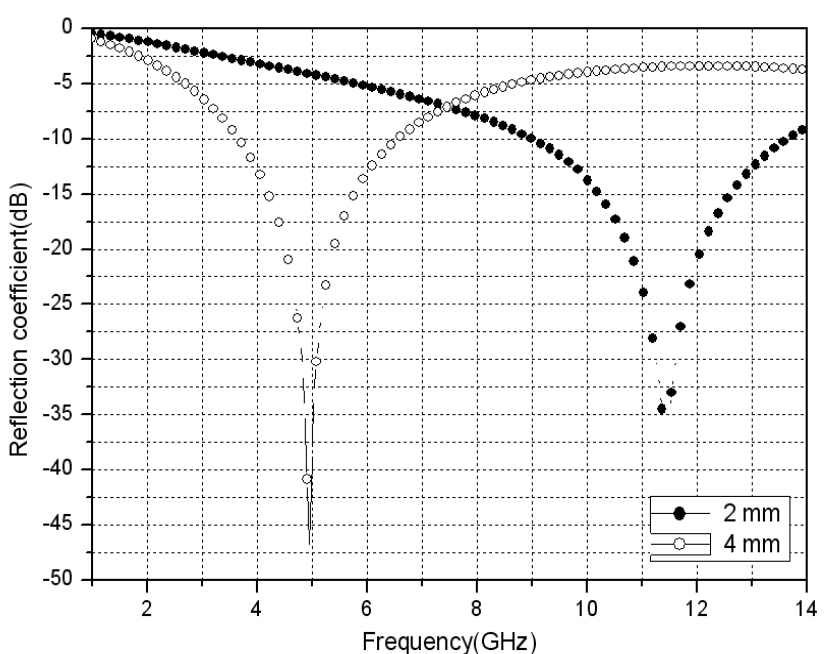

(a) Reflection coefficients of samples with the fixed ratio of flaked sendust:cPE=60:40 wt.\%

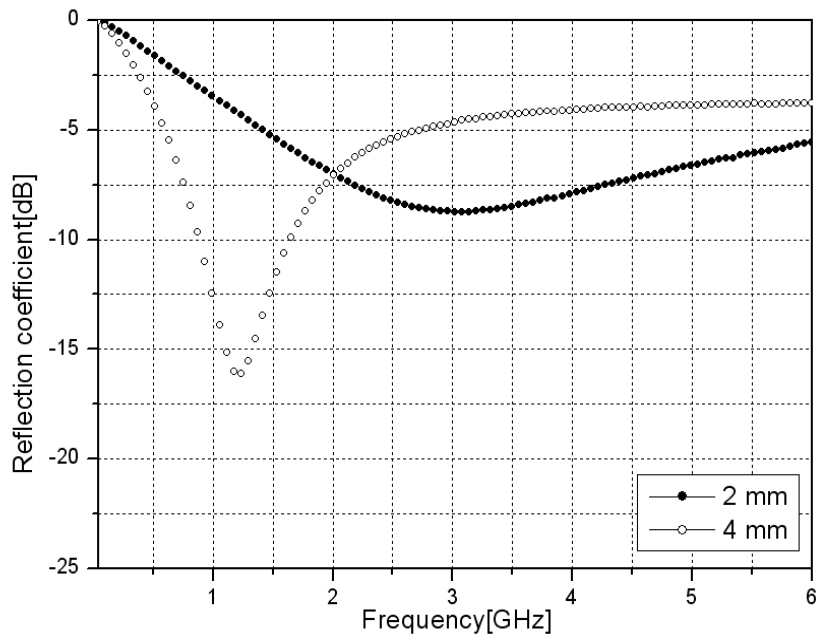

(b) Reflection coefficients of samples with the fixed ratio of flaked sendust:CPE=70:30 wt.\%

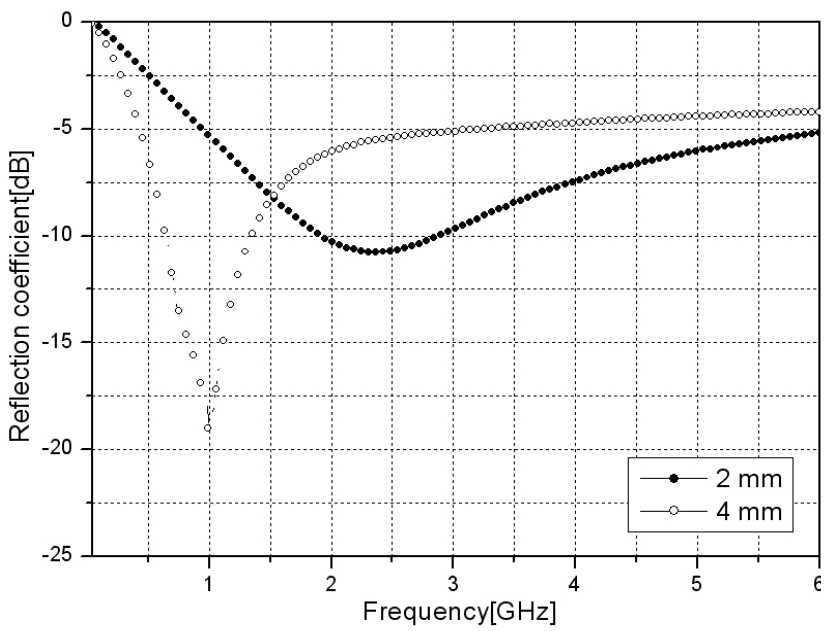

(c) Reflection coefficients of samples with the fixed ratio of flaked sendust:CPE=80:20 wt.\%

Fig. 7. Reflection coefficients of samples with different composition ratio (thickness: 2, $4 \mathrm{~mm}$ ).

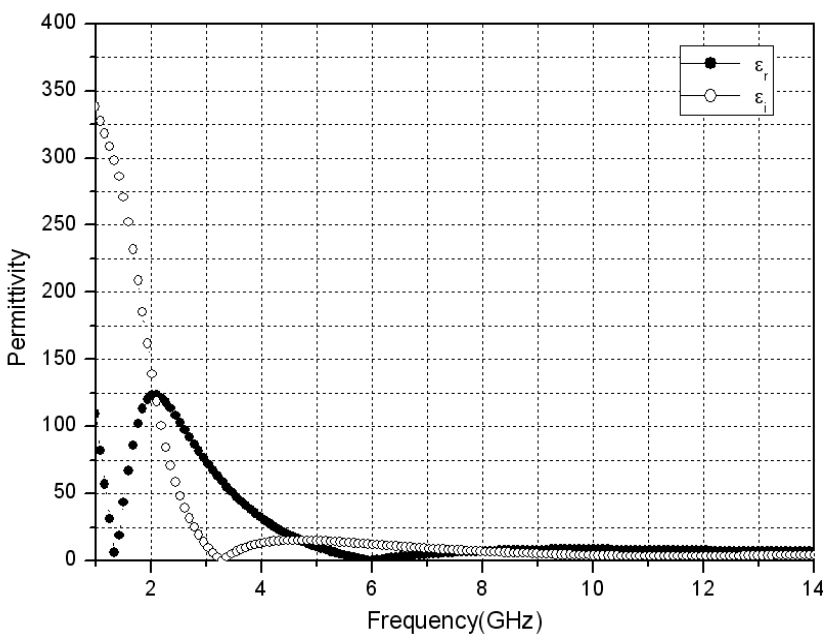

(a) Relative permittivity of samples with the fixed ratio of flaked sendust: $\mathrm{CPE}=60: 40$ wt. $\%$.

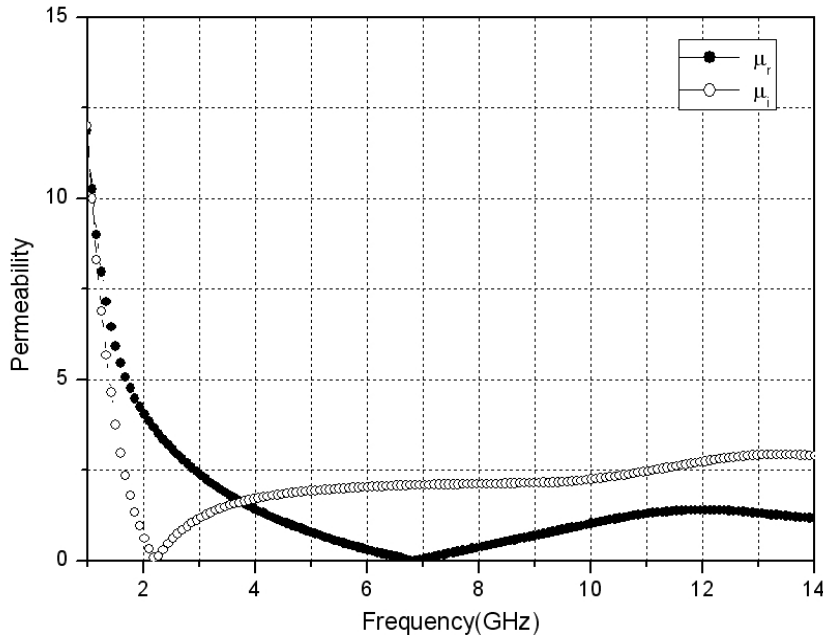

(b) Relative permeability of samples with the fixed ratio of flaked sendust:CPE=60:40 wt. $\%$

Fig. 8. Material constants of the samples by flaked sendust: $\mathrm{CPE}=60: 40$ wt. $\%$.

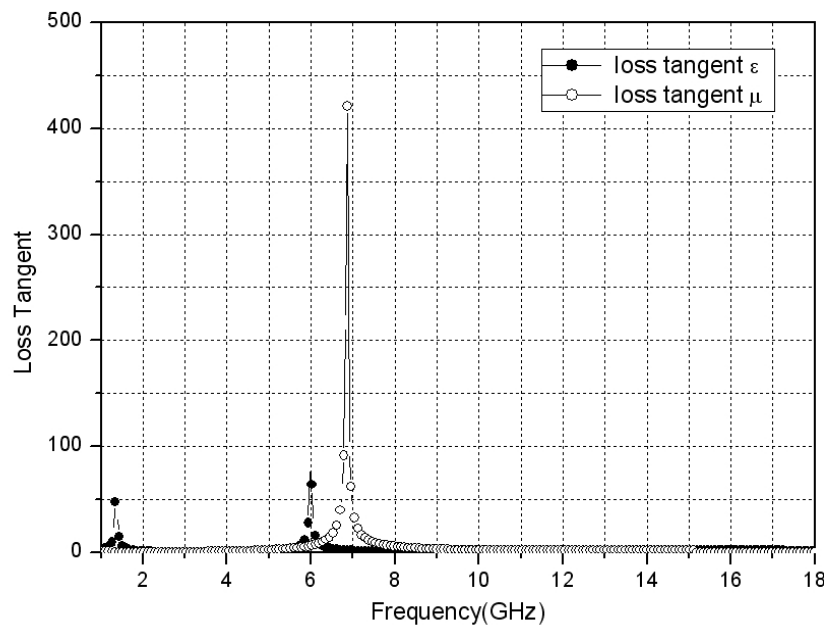

Fig. 9. Loss tangent of samples with the mixing ratio of flaked sendust: $\mathrm{CPE}=60: 40$ wt. $\%$. 


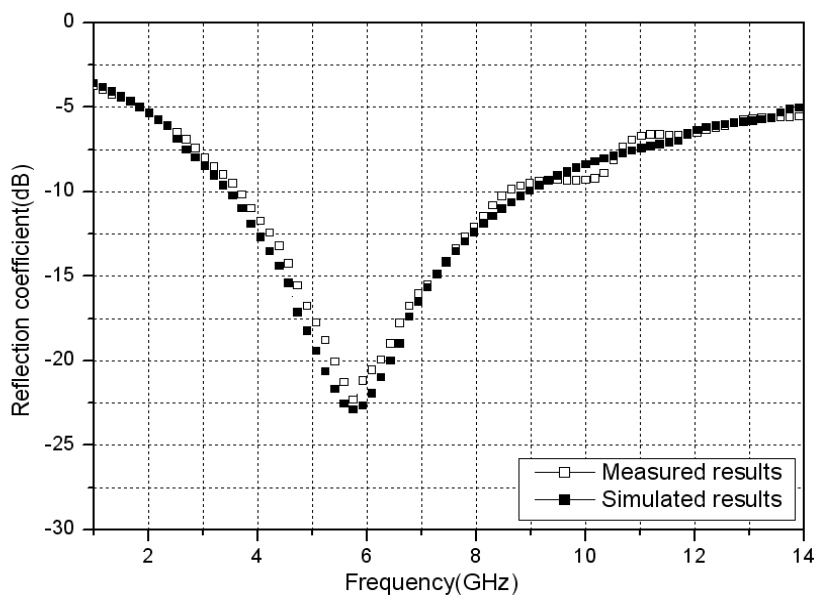

Fig. 10. Comparisons of simulated and measured results in the case of vertical incidence wave.

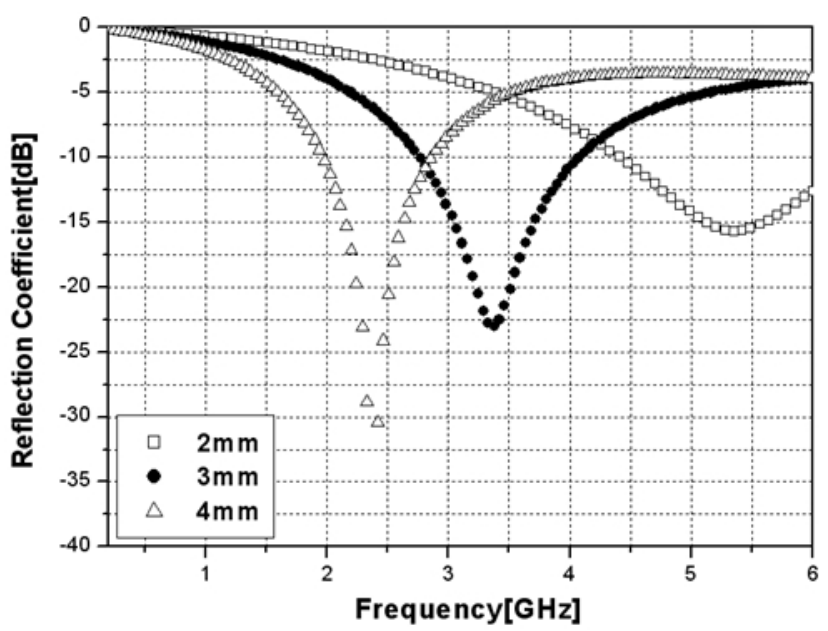

Fig. 11. Reflection coefficients of a conventional sample (Amorphous:CPE=60:40 wt.\%).

$=60: 40$ wt. $\%$.

\section{Conclusion}

In this paper, we investigated EM wave absorber for ETC system. The EM wave absorber samples were made of flaked sendust as a magnetic material and CPE as binder. The absorption abilities of the samples were measured by a vector network analyzer. We compared the absorption abilities of several samples. These material properties were calculated from the S-parameters. The best EM wave absorber was designed by using the material property and changing thickness at $5.8 \mathrm{GHz}$ for ETC system.

The optimized composition ratio of absorbing materials was derived as flaked sendust: $\mathrm{CPE}=60: 40 \mathrm{wt} . \%$, and absorption ability of the EM wave absorber with thickness of $2.7 \mathrm{~mm}$ showed absorption ability of $22.5 \mathrm{~dB}$ for normal incidence wave. Therefore, it was confirmed that the newly developed absorber can be used for in ETC system.

This work was supported by the program of the Academic Research \& Development from the Korea Sanhak-Corporation Foundation.

\section{References}

[1] K. Haneda, J. Takada, T. Iwata, Y. Wakinaka, and T. Kunishima, "Experimental determination of propagation paths for the ETC system - Equipment development and field test," Trans. IEICE, vol. E87-A, no. 11, pp. 3008-3015, Nov. 2004.

[2] M. Hanazawa, O. Hashimoto, and K. Wada, "New wave absorber using resistive film for ETC system," EMC Europe 2002 Int Symp. Dig., vol. 2, pp. 703705, Sep. 2002.

[3] T. Doi, N. Tashiro, T. Inoue, A. Fujita, and O. Hashimoto, "A study on a wave absorber using anisotropic carbon for ETC application," Proc, of 2005 IEEE AP-S International Symposium on Antennas and Propagation and USNC/URSI National Radio Science Meeting, no. 61-7, Jul. 2005.

[4] K. Matsumoto, Y. Miura, T. Ozawa, O. Hashimoto, and O. Okada, "Wave absorber with circular lattice using metal mesh for improving ETC environment," EMC Zurich 2007. 18th International Zurich Symposium, no. 24-28, pp. 233-236, Sep. 2007.

[5] Dae-Hun Kim, Dong Il Kim, Chang-Mook Choi, and Jun-Young Son, "A study on design and fabrication of complex type EM wave absorber with super wide-band characteristics," International Joural of $\mathrm{Na}$ vigation and Port Research, vol. 30, no. 2, pp. 161166, Mar. 2006.

[6] Chang-Mook Choi, Dong Il Kim, Rui Li, and Kwang-Soob Ko, "Development of the electromagnetic wave absorber for $94 \mathrm{GHz}$ radar sensors using permalloy," Internat. J. KIMICS, vol. 4, no. 3, pp. 1-4, Sep. 2006.

[7] Sang-Hyun Moon, Seung-Jae Shin, Jae-Man Song, Dong Il Kim, and Ki-Man Kim, "A study on the development of ferrite EM wave absorbers for $\mathrm{GHz}$ frequency band," Korea Electromagnetic Engineering Soc., vol. 14, no. 12, pp. 1329-1334, Dec. 2003.

[8] Y. Naito, Electromagnetic Wave Absorbers, Tokyo : New Ohm, 1987.

[9] T. Weiland, "A discretization method for the solution of maxwell's equations for six-component fields," Electronics and Communication(AEU), vol. 31, no. 3, pp. 116-120, 1977. 


\section{Dong Il Kim}

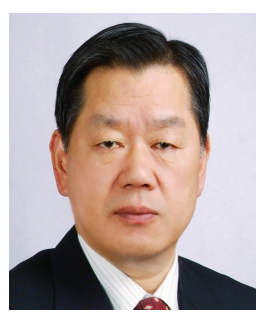

was born in Daejeon, Korea. He received the B.E. and M.E. degrees in nautical science and electronic communications from Korea Maritime University in 1975 and 1977, respectively. He received the Ph.D. degree in electronics from the Tokyo Institute of Technology in 1984. Currently, he is a professor of the Dept. of Radio Sciences \& Engineering at Korea Maritime University. He was in charge of the president of the Korean Institute of Electromagnetic Engineering Society (KIEES) and the IEEE Korea Chapter Chairman from 2002 to 2003. His research interests include the design of microwave circuits, the development of EM absorbers, and EMC/EMI countermeasures.

He received the Academy Industry Cooperation (A-I-C) Award from the Korea A-I-C. Foundation in 1990, the Treatise Awards from the Korea Electromagnetic Engineering Society and the Korea Institute of Navigation in 1993 and 1998, the Korea President's Award from the Promotion of Science and Technology in 1995, and the Great Award of Education in Korea in 2010, respectively. He is a member of IEEE, the Institute of Electronics, Information and Communications of Japan, the KIEES, the IEEK, and the KICS of Korea.

\section{Dong Soo Choi}

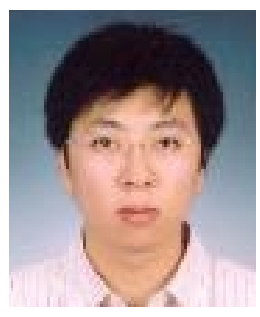

received the B.E. and M.E. degree from Korea Maritime University in 2009 and 2011. He is currently working toward the $\mathrm{Ph} . \mathrm{D}$. degree in department of radio communication engineering at Korea Maritime University. His research interests include the development of EM wave absorber, EMI/EMC.

\section{Dong Han Choi}

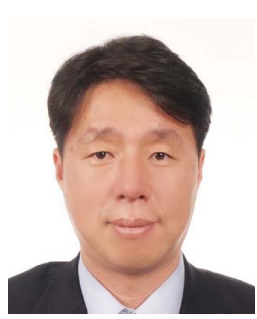

received the B.E. degree from Kyungsung University in 1998, his M.E degree in Pukyong National University 2000, and the Ph.D. degree in Bradio sciences $\&$ engineering from Korea Maritime University in 2006. His research interests include the EMI/EMC.

\section{Do Yeol Kim}

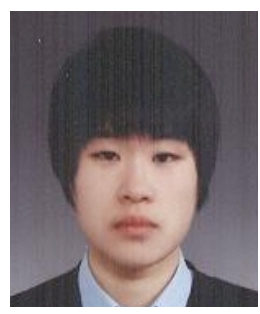

received the B.E. degree from Korea Maritime University in 2012. He is currently working toward the M.E. degree in the department of radio communication engineering at Korea Maritime University. His research interests include the development of EM wave absorber and EMI/ EMC. 